\title{
Bartholdi Zeta Functions of Fractal Graphs
}

\author{
Iwao Sato \\ Oyama National College of Technology, \\ Oyama, Tochigi 323-0806, Japan \\ e-mail: isato@oyama-ct.ac.jp
}

Submitted: Aug 12, 2008; Accepted: Feb 18, 2009; Published: Feb 27, 2009

Mathematical Subject Classification: 05C50, 05C25, 05C10, 15A15

\begin{abstract}
Recently, Guido, Isola and Lapidus [11] defined the Ihara zeta function of a fractal graph, and gave a determinant expression of it. We define the Bartholdi zeta function of a fractal graph, and present its determinant expression.
\end{abstract}

\section{Introduction}

Zeta functions of graphs started from $p$-adic Selberg zeta functions of discrete groups by Ihara [14]. At the beginning, Serre [20] pointed out that the Ihara zeta function is the zeta function of a regular graph. In [14], Ihara showed that their reciprocals are explicit polynomials. A zeta function of a regular graph $G$ associated to a unitary representation of the fundamental group of $G$ was developed by Sunada [22,23]. Hashimoto [13] treated multivariable zeta functions of bipartite graphs. Bass [3] generalized Ihara's result on zeta functions of regular graphs to irregular graphs. Various proofs of Bass' theorem were given by Stark and Terras [21], Kotani and Sunada [15] and Foata and Zeilberger [5].

Bartholdi [2] extended a result by Grigorchuk [7] relating cogrowth and spectral radius of random walks, and gave an explicit formula determining the number of bumps on paths in a graph. Furthermore, he presented the "circuit series" of the free products and the direct products of graphs, and obtained a generalized form "Bartholdi zeta function" of the Ihara(-Selberg) zeta function.

All graphs in this paper are assumed to be simple. Let $G$ be a connected graph with vertex set $V(G)$ and edge set $E(G)$, and let $R(G)=\{(u, v),(v, u) \mid u v \in E(G)\}$ be the set of oriented edges (or arcs) $(u, v),(v, u)$ directed oppositely for each edge $u v$ of $G$. For $e=(u, v) \in R(G), u=o(e)$ and $v=t(e)$ are called the origin and the terminal of $e$, respectively. Furthermore, let $e^{-1}=(v, u)$ be the inverse of $e=(u, v)$.

A path $P$ of length $n$ in $G$ is a sequence $P=\left(e_{1}, \cdots, e_{n}\right)$ of $n$ arcs such that $e_{i} \in R(G)$, $t\left(e_{i}\right)=o\left(e_{i+1}\right)(1 \leq i \leq n-1)$. If $e_{i}=\left(v_{i-1}, v_{i}\right), 1 \leq i \leq n$, then we also denote $P$ by $\left(v_{0}, v_{1}, \cdots, v_{n}\right)$. Set $|P|=n, o(P)=o\left(e_{1}\right)$ and $t(P)=t\left(e_{n}\right)$. Also, $P$ is called an 
$(o(P), t(P))$-path. A $(v, w)$-path is called a $v$-closed path if $v=w$. The inverse of a closed path $C=\left(e_{1}, \cdots, e_{n}\right)$ is the closed path $C^{-1}=\left(e_{n}^{-1}, \cdots, e_{1}^{-1}\right)$.

We say that a path $P=\left(e_{1}, \cdots, e_{n}\right)$ has a backtracking or a bump at $t\left(e_{i}\right)$ if $e_{i+1}^{-1}=e_{i}$ for some $i(1 \leq i \leq n-1)$. A path without backtracking is called proper. Let $B^{r}$ be the closed path obtained by going $r$ times around a closed path $B$. Such a closed path is called a multiple of $B$. Multiples of a closed path without bumps may have a bump. Such a closed path is said to have a tail. If its length is $n$, then the closed path can be written as

$$
\left(e_{1}, \cdots, e_{k}, f_{1}, f_{2}, \cdots, f_{n-2 k}, e_{k}^{-1}, \cdots, e_{1}^{-1}\right),
$$

where $\left(f_{1}, f_{2}, \cdots, f_{n-2 k}\right)$ is a closed path. A closed path is called reduced if $C$ has no backtracking nor tail. Furthermore, a closed path $C$ is primitive if it is not a multiple of a strictly shorter closed path. Let $\mathcal{C}$ be the set of closed paths. Furthermore, let $\mathcal{C}^{\text {nontail }}$ and $\mathcal{C}^{\text {tail }}$ be the set of closed paths without tail, and closed paths with tail, respectively. Note that $\mathcal{C}=\mathcal{C}^{\text {nontail }} \cup \mathcal{C}^{\text {tail }}$ and $\mathcal{C}^{\text {nontail }} \cap \mathcal{C}^{\text {tail }}=\phi$.

We introduce an equivalence relation between closed paths. Two closed paths $C_{1}=$ $\left(e_{1}, \cdots, e_{m}\right)$ and $C_{2}=\left(f_{1}, \cdots, f_{m}\right)$ are called equivalent if there exists an integer $k$ such that $f_{j}=e_{j+k}$ for all $j$, where the subscripts are read modulo $n$. The inverse of $C$ is not equivalent to $C$ if $|C| \geq 3$. Let $[C]$ be the equivalence class which contains a closed path $C$. Also, $[C]$ is called a cycle.

Let $\mathcal{K}$ be the set of cycles of $G$. Denote by $\mathcal{R}, \mathcal{P} \subset \mathcal{R}$ and $\mathcal{P} \mathcal{K} \subset \mathcal{K}$ the set of reduced cycles, primitive, reduced cycles and primitive cycles of $G$, respectively. Also, primitive, reduced cycles are called prime cycles. Let $\mathcal{C}_{m}, \mathcal{C}_{m}^{\text {nontail }}, \mathcal{C}_{m}^{\text {tail }}, \mathcal{K}_{m}$ and $\mathcal{P} \mathcal{K}_{m}$ be the subset of $\mathcal{C}, \mathcal{C}^{\text {nontail }}, \mathcal{C}^{\text {tail }}, \mathcal{K}$ and $\mathcal{P} \mathcal{K}$ consisting of elements with length $m$, respectively. Note that each equivalence class of primitive, reduced closed paths of a graph $G$ passing through a vertex $v$ of $G$ corresponds to a unique conjugacy class of the fundamental group $\pi_{1}(G, v)$ of $G$ at $v$.

The Ihara zeta function of a graph $G$ is a function of a complex variable $t$ with $|t|$ sufficiently small, defined by

$$
\mathbf{Z}(G, t)=\mathbf{Z}_{G}(t)=\prod_{[C] \in \mathcal{P}}\left(1-t^{|C|}\right)^{-1}
$$

where $[C]$ runs over all prime cycles of $G$.

Let $G$ be a connected graph with $n$ vertices $v_{1}, \cdots, v_{n}$. The adjacency matrix $\mathbf{A}=$ $\mathbf{A}(G)=\left(a_{i j}\right)$ is the square matrix such that $a_{i j}=1$ if $v_{i}$ and $v_{j}$ are adjacent, and $a_{i j}=0$ otherwise. The degree of a vertex $v_{i}$ of $G$ is defined by $\operatorname{deg} v_{i}=\operatorname{deg}_{G} v_{i}=\mid\left\{v_{j} \mid v_{i} v_{j} \in\right.$ $E(G)\} \mid$. If $\operatorname{deg}_{G} v=k$ (constant) for each $v \in V(G)$, then $G$ is called $k$-regular.

Ihara [14] showed that the reciprocal of the Ihara zeta function of a regular graph is an explicit polynomial. The Ihara zeta function of a regular graph has the following three properties: the rationality; the functional equations; the analogue of the Riemann hypothesis(see [24]). The analogue of the Riemann hypothesis for the zeta function of a graph is given as follows: Let $G$ be any connected $(q+1)$-regular $\operatorname{graph}(q>1)$ and $s=\sigma+i t(\sigma, t \in \mathbf{R})$ a complex number. If $\mathbf{Z}_{G}\left(q^{-s}\right)=0$ and $\operatorname{Re} s \in(0,1)$, then $\operatorname{Re} s=\frac{1}{2}$. 
A connected $(q+1)$-regular graph $G$ is called a Ramanujan graph if for all eigenvalues $\lambda$ of the adjacency matrix $\mathbf{A}(G)$ of $G$ such that $\lambda \neq \pm(q+1)$, we have $|\lambda| \leq 2 \sqrt{q}$. This definition was introduced by Lubotzky, Phillips and Sarnak [16]. For a connected $(q+1)$-regular graph $G, \mathbf{Z}_{G}\left(q^{-s}\right)$ satisfies the Riemann hypothesis if and only if $G$ is a Ramanujan graph.

Hashimoto [13] treated multivariable zeta functions of bipartite graphs. Bass [3] generalized Ihara's result on the Ihara zeta function of a regular graph to an irregular graph, and showed that its reciprocal is a polynomial.

Theorem 1 (Bass) Let $G$ be a connected graph. Then the reciprocal of the Ihara zeta function of $G$ is given by

$$
\mathbf{Z}(G, t)^{-1}=\left(1-t^{2}\right)^{r-1} \operatorname{det}\left(\mathbf{I}-t \mathbf{A}(G)+t^{2}(\mathbf{D}-\mathbf{I})\right),
$$

where $r$ is the Betti number of $G$, and $\mathbf{D}=\left(d_{i j}\right)$ is the diagonal matrix with $d_{i i}=\operatorname{deg} v_{i}$ and $d_{i j}=0, i \neq j,\left(V(G)=\left\{v_{1}, \cdots, v_{n}\right\}\right)$.

Stark and Terras [21] gave an elementary proof of Theorem 1, and discussed three different zeta functions of any graph. Various proofs of Bass' theorem were known. Kotani and Sunada [15] proved Bass' theorem by using the property of the Perron operator. Foata and Zeilberger [5] presented a new proof of Bass' theorem by using the algebra of Lyndon words.

Let $G$ be a connected graph. Then the bump count $b c(P)$ of a path $P$ is the number of bumps in $P$. Furthermore, the cyclic bump count $c b c(C)$ of a closed path $C=\left(e_{1}, \cdots, e_{n}\right)$ is

$$
c b c(C)=\left|\left\{i=1, \cdots, n \mid e_{i}=e_{i+1}^{-1}\right\}\right|,
$$

where $e_{n+1}=e_{1}$. An equivalence class of primitive closed paths in $G$ is called a primitive cycle. Then the Bartholdi zeta function of $G$ is a function of complex variables $u, t$ with $|u|,|t|$ sufficiently small, defined by

$$
\zeta_{G}(u, t)=\zeta(G, u, t)=\prod_{[C] \in \mathcal{P} \mathcal{K}}\left(1-u^{c b c(C)} t^{|C|}\right)^{-1},
$$

where $[C]$ runs over all primitive cycles of $G$.

If $u=0$, then the Bartholdi zeta function of $G$ is the Ihara zeta function of $G$. Because the Bartholdi zeta function $\zeta(G, u, t)$ of a graph is divided into two parts concerned with primitive, non-reduced cycles and primitive, reduced cycles (i.e., prime cycles) of $G$, respectively:

$$
\zeta(G, u, t)=\prod_{[C] \in \mathcal{P} \mathcal{K} \backslash \mathcal{P}}\left(1-u^{c b c(C)} t^{|C|}\right)^{-1} \times \prod_{[C] \in \mathcal{P}}\left(1-t^{|C|}\right)^{-1} .
$$

By substituting $u=0$, we obtain

$$
\zeta(G, 0, t)=1 \cdot \prod_{[C] \in \mathcal{P}}\left(1-t^{|C|}\right)^{-1}=\mathbf{Z}(G, t) .
$$


Let $n$ and $m$ be the number of vertices and unoriented edges of $G$, respectively. Then two $2 m \times 2 m$ matrices $\mathbf{B}=\left(\mathbf{B}_{e, f}\right)_{e, f \in R(G)}$ and $\mathbf{J}=\left(\mathbf{J}_{e, f}\right)_{e, f \in R(G)}$ are defined as follows:

$$
\mathbf{B}_{e, f}=\left\{\begin{array}{ll}
1 & \text { if } t(e)=o(f), \\
0 & \text { otherwise }
\end{array}, \mathbf{J}_{e, f}= \begin{cases}1 & \text { if } f=e^{-1} \\
0 & \text { otherwise. }\end{cases}\right.
$$

Bartholdi [2] presented a determinant expression for the Bartholdi zeta function of a graph.

Theorem 2 (Bartholdi) Let $G$ be a connected graph with $n$ vertices and $m$ unoriented edges. Then the reciprocal of the Bartholdi zeta function of $G$ is given by

$$
\begin{aligned}
\zeta(G, u, t)^{-1} & =\operatorname{det}\left(\mathbf{I}_{2 m}-(\mathbf{B}-(1-u) \mathbf{J}) t\right) \\
& =\left(1-(1-u)^{2} t^{2}\right)^{m-n} \operatorname{det}\left(\mathbf{I}-t \mathbf{A}(G)+(1-u)(\mathbf{D}-(1-u) \mathbf{I}) t^{2}\right) .
\end{aligned}
$$

In the case of $u=0$, Theorem 2 implies Theorem 1 .

The Ihara zeta function of a finite graph was extended to an infinite graph in $[3,4,8,9$, $10,11]$, and those determinant expressions were presented. Bass [3] defined the zeta function for a pair of a tree $X$ and a countable group $\Gamma$ which acts discretely on $X$ with quotient being a graph of finite groups. Clair and Mokhtari-Sharghi [4] extended Ihara zeta functions to infinite graphs on which a group $\Gamma$ acts isomorphically and with finite quotient. In [8], Grigorchuk and Żuk defined zeta functions of infinite discrete groups, and of some class of infinite periodic graphs.

Guido, Isola and Lapidus [9] defined the Ihara zeta function of a periodic simple graph(i.e., an infinite graph). Let $G=(V(G), E(G))$ be a simple graph which is (countable and) uniformly locally finite, and let $\Gamma$ be a countable discrete subgroup of automorphisms of $G$, which acts freely on $G$, and with finite quotient $B=G / \Gamma$. Then the Ihara zeta function of a periodic simple graph is defined as follows:

$$
\mathbf{Z}_{G, \Gamma}(t)=\prod_{[C]_{\Gamma} \in[\mathcal{P}]_{\Gamma}}\left(1-t^{|C|}\right)^{-1 /\left|\Gamma_{C}\right|}
$$

where $[C]_{\Gamma}$ runs over all $\Gamma$-equivalence classes of prime cycles in $G$.

Guido, Isola and Lapidus [9] presented a determinant expression for the Ihara zeta function of a periodic simple graph by using Stark and Terras' method [21].

\section{Theorem 3 (Guido, Isola and Lapidus)}

$$
\mathbf{Z}_{G, \Gamma}(t)=\left(1-t^{2}\right)^{-(m-n)} \operatorname{det}_{\Gamma}\left(\mathbf{I}-t \mathbf{A}(G)+(\mathbf{D}-\mathbf{I}) t^{2}\right)^{-1},
$$

where $m=|E(B)|, n=|V(B)|$ and $\operatorname{det}_{\Gamma}$ is a determinant for bounded operators belonging to a von Neumann algebra with a finite trace.

Also, Guido, Isola and Lapidus [10] presented a determinant expression for the Ihara zeta function of a periodic graph by using Bass' method [3]. Furthermore, Guido, Isola 
and Lapidus [11] generalized the results of $[9,10]$ to a fractal graph. In [11], they defined the Ihara zeta function of a fractal graph and gave its determinant expression.

In this paper, we define the Bartholdi zeta function of a fractal graph, and present its determinant expression. The proof is an analogue of the method of Guido, Isola and Lapidus [11], and Mizuno and Sato's method [17]. In Section 2, we give a short review on a fractal graph. In Section 3, we present some combinatorial properties on closed paths of a fractal graph. In Section 4, we define the Bartholdi zeta function of a fractal graph, and show that it is holomorphic. In Section 5, we review a determinant for bounded operators acting on an infinite dimensional Hilbert space and belonging to a von Neumann algebra with a finite trace. In Section 6, we present a determinant expression for the Bartholdi zeta function of a fractal graph.

\section{$2 \quad$ Fractal graphs}

Let $G=(V(G), E(G))$ be countable and connected. We assume that $G$ has bounded degree, i.e., $d=\sup _{v \in V(G)} \operatorname{deg}_{G} v<\infty$ (see $\left.[18,19]\right)$. For two vertices $v, w \in V(G)$, the distance $d(v, w)$ between $v$ and $w$ is defined as the length of the shortest path between $v$ and $w$. For $v \in V(G)$ and $r \in \mathbf{N}$, let $B_{r}(v)=\{w \in V(G) \mid d(v, w) \leq r\}$. For $\Omega \subset V(G)$, let $B_{r}(\Omega)=\cup_{v \in \Omega} B_{r}(v)$.

A bounded operator $A$ on $\ell^{2}(V(G))$ has finite propagation $r=r(A) \geq 0$ if, for all $v \in V(G), \operatorname{supp}(A v) \subset B_{r}(v)$ and $\operatorname{supp}\left(A^{*} v\right) \subset B_{r}(v) \mathrm{S}$, where $A^{*}$ is the Hilbert space adjoint of $A$. Let $B\left(\ell^{2}(V(G))\right)$ be the set of bounded operators on $\ell^{2}(V(G))$. Note that finite propagation operators forms a $*$-algebra.

A local isomorphim of the graph $G$ is a triple $(s(\gamma), r(\gamma), \gamma)$, where $s(\gamma), r(\gamma)$ are subgraphs of $G$ and $\gamma: s(\gamma) \longrightarrow r(\gamma)$ is a graph isomorphism. The local isomorpism $\gamma$ defines a partial isometry $U(\gamma): \ell^{2}(V(G)) \longrightarrow \ell^{2}(V(G))$, by setting

$$
U(\gamma)(v):= \begin{cases}\gamma(v) & \text { if } v \in V(s(\gamma)) \\ 0 & \text { otherwise }\end{cases}
$$

and extending by linearity.

An operator $T \in B\left(\ell^{2}(V(G))\right)$ is called geometric if there exists $r \in \mathbf{N}$ such that $T$ has finite propagation $r$ and, for any local isomorphism $\gamma$, any vertex $v \in V(G)$ such that $B_{r}(v) \subset s(\gamma)$ and $B_{r}(\gamma v) \subset r(\gamma)$, one has

$$
T U(\gamma) v=U(\gamma) T v, T^{*} U(\gamma) v=U(\gamma) T^{*} v
$$

The adjacencey matrix $\mathbf{A}(G)=\left(a_{v w}\right)$ and the degree matrix $\mathbf{D}(G)=\left(d_{v w}\right)$ are defined by

$$
a_{v w}:= \begin{cases}1 & \text { if }(v, w) \in R(G) \\ 0 & \text { otherwise }\end{cases}
$$

and

$$
d_{v w}:= \begin{cases}\operatorname{deg}_{G} v & \text { if } v=w \\ 0 & \text { otherwise }\end{cases}
$$


For a subgraph $K$ of $G$, the frontier $\mathcal{F}(K)$ is the family of vertices in $V(K)$ having distance 1 from the complement of $V(K)$ in $V(G)$. A countably infinite graph $G$ with bounded degree is amenable if it has an amenable exhaustion, i.e., an increasing family of finite subgraphs $\left\{K_{n}\right\}_{n \in \mathbf{N}}$ such that $\cup_{n \in \mathbf{N}} K_{n}=G$ and

$$
\frac{\left|\mathcal{F}\left(K_{n}\right)\right|}{\left|V\left(K_{n}\right)\right|} \longrightarrow 0 \text { as } n \rightarrow \infty \text {. }
$$

A countably infinite graph $G$ with bounded degree is called self-similar or fractal if it has an amenable exhaustion $\left\{K_{n}\right\}$ such that the following conditions (i) and (ii) hold(see $[1,12])$ :

(i) For every $n \in \mathbf{N}$, there is a finite set $\mathcal{I}(n . n+1)$ of local isomorphisms such that, for all $\gamma \in \mathcal{I}(n, n+1)$, one has $s(\gamma)=K_{n}$,

$$
\bigcup_{\gamma \in \mathcal{I}(n, n+1)} \gamma\left(K_{n}\right)=K_{n+1},
$$

and moreover, if $\gamma, \gamma^{\prime} \in \mathcal{I}(n, n+1)$ with $\gamma \neq \gamma^{\prime}$,

$$
V\left(\gamma K_{n}\right) \cap V\left(\gamma^{\prime} K_{n}\right)=\mathcal{F}\left(\gamma K_{n}\right) \cap \mathcal{F}\left(\gamma^{\prime} K_{n}\right) .
$$

(ii) Let $\mathcal{I}(n, m)(n<m)$ be the set of all admissible products $\gamma=\gamma_{m-1} \cdots \gamma_{n}, \gamma_{i} \in$ $\mathcal{I}(i, i+1)$, where "admissble" means that, for each term of the product, the range of $\gamma_{i}$ is contained in the source of $\gamma_{i+1}$. Also, let $\mathcal{I}(n, n)=\left\{i d_{K_{n}}\right\}$, and $\mathcal{I}(n)=\cup_{m \geq n} \mathcal{I}(n, m)$.

We define the $\mathcal{I}$-invariant frontier of $K_{n}$ :

$$
\mathcal{F}_{\mathcal{I}}\left(K_{n}\right)=\bigcup_{\gamma \in \mathcal{I}(n)} \gamma^{-1} \mathcal{F}\left(\gamma K_{n}\right) .
$$

and we require that

$$
\frac{\left|\mathcal{F}_{\mathcal{I}}\left(K_{n}\right)\right|}{\left|V\left(K_{n}\right)\right|} \longrightarrow 0 \text { as } n \rightarrow \infty .
$$

Let $\mathcal{I}$ be the family of all local isomorphisms which can be written as admissible products $\gamma_{1}^{\epsilon_{1}} \gamma_{2}^{\epsilon_{2}} \cdots \gamma_{k}^{\epsilon_{k}}$, where $\gamma_{i} \in \cup_{n \in \mathbf{N}} \mathcal{I}(n), \epsilon_{i}=1,-1$ for $i=1, \ldots, k$ and $k \in \mathbf{N}$.

A trace on the algebra of geometric operators on a fractal graph is constructed as follows(see [11]):

Theorem 4 (Guido, Isola and Lapidus) Let $G$ be a fractal graph, and $\mathcal{A}(G)$ the *algebra defined as the norm closure of the *-algebra of geometric operators. Then, on $\mathcal{A}(G)$, there is a well-defined faithful trace state $\operatorname{Tr}_{\mathcal{I}}$ given by

$$
\operatorname{Tr}_{\mathcal{I}}(T)=\lim _{n} \frac{\operatorname{Tr}\left(P\left(K_{n}\right) T\right)}{\operatorname{Tr}\left(P\left(K_{n}\right)\right)},
$$

where $P\left(K_{n}\right)$ is the orthogonal projection of $\ell^{2}(V(G))$ onto its closed subspace $\ell^{2}\left(V\left(K_{n}\right)\right)$. 
We use the following result by Guido, Isola and Lapidus [11].

Proposition 1 (Guido, Isola and Lapidus) Let $G$ be a connected fractal graph with bounded degree $d=\sup _{v \in V(G)} \operatorname{deg}_{G} v<\infty$. Furthermore, let $\left\{K_{n}\right\}$ be an amenable exhaustion of $G$ such that satisfies the conditions (i) and (ii) in the definition of a fractal graph. Let $\Omega$ be any finite subset of $V(G)$. Then the following results hold:

1. For any $r \in \mathbf{N}$,

$$
\left|B_{r}(\Omega)\right| \leq|\Omega|(d+1)^{r} .
$$

2. Let $\Omega_{n, r}=V\left(K_{n}\right) \backslash B_{r}\left(\mathcal{F}_{\mathcal{I}}\left(K_{n}\right)\right)$. Then, for $n \leq m$,

$$
\left|\mathcal{I}(n, m)\left\|\Omega_{n, r}|\leq| V\left(K_{m}\right)|\leq| \mathcal{I}(n, m)\right\| V\left(K_{n}\right)\right|
$$

3. Let

$$
\epsilon_{n}=\frac{\left|\mathcal{F}_{\mathcal{I}}\left(K_{n}\right)\right|}{\left|V\left(K_{n}\right)\right|}
$$

where $\epsilon_{n} \rightarrow 0$ as $n \rightarrow \infty$ by the definition of a fractal graph. Furthermore, let $\epsilon_{n}(d+1)^{r} \leq 1 / 2$ for all $n>n_{0}$. Then

$$
0 \leq \frac{|\mathcal{I}(n, m)|\left|V\left(K_{n}\right)\right|}{\left|V\left(K_{m}\right)\right|}-1 \leq 2 \epsilon_{n}(d+1)^{r} \leq 1
$$

\section{Closed paths in a fractal graph}

Let $G$ be a connected fractal graph. Furthermore, let $\left\{K_{n}\right\}$ be an amenable exhaustion of $G$ such that satisfies the conditions (i) and (ii) in the definition of a fractal graph. Let $0<u<1$. For $s \geq 1$, the matrix $\mathbf{A}_{s}=\left(\left(\mathbf{A}_{s}\right)_{i, j}\right)_{v_{i}, v_{j} \in V(G)}$ is defined as follows:

$$
\left(\mathbf{A}_{s}\right)_{i, j}=\sum_{P} u^{b c(P)}
$$

where $\left(\mathbf{A}_{s}\right)_{i, j}$ is the $(i, j)$-component of $\mathbf{A}_{s}$, and $P$ runs over all paths of length $s$ from $v_{i}$ to $v_{j}$ in $G$. Note that $\mathbf{A}_{1}=\mathbf{A}(G)$. Furthermore, let $\mathbf{A}_{0}=\mathbf{I}$.

Lemma 1 Put $\mathbf{Q}=\mathbf{D}-\mathbf{I}$. Then

$$
\mathbf{A}_{2}=\left(\mathbf{A}_{1}\right)^{2}-(1-u) \mathbf{D}=\left(\mathbf{A}_{1}\right)^{2}-(1-u)(\mathbf{Q}+\mathbf{I})
$$

and

$$
\mathbf{A}_{s}=\mathbf{A}_{s-1} \mathbf{A}_{1}-(1-u) \mathbf{A}_{s-2}(\mathbf{Q}+u \mathbf{I}) \text { for } s \geq 3
$$


Proof. The first formula is clear. We prove the second formula. The proof is an analogue of the proof of Lemma 1 in [21].

We count the paths of length $s$ from $v_{i}$ to $v_{k}$ in $G$. Let $s \geq 3$ and $\mathbf{A}(G)=\left(\mathbf{A}_{i, j}\right)$. Then the sum $\sum_{j}\left(\mathbf{A}_{s-1}\right)_{i, j} \mathbf{A}_{j, k}$ counts three types of paths $P, Q, R$ in $G$ as follows:

$$
\begin{aligned}
& P=\left(e_{1}, \cdots, e_{s-1}, e_{s}\right), e_{s} \neq e_{s-1}^{-1}, e_{s}=\left(v_{j}, v_{k}\right), \\
& Q=\left(e_{1}, \cdots, e_{s-2}, e_{s-1}, e_{s}\right), e_{s-1} \neq e_{s-2}^{-1}, e_{s}=e_{s-1}^{-1}=\left(v_{j}, v_{k}\right), \\
& R=\left(e_{1}, \cdots, e_{s-2}, e_{s-1}, e_{s}\right), e_{s-2}=e_{s-1}^{-1}=e_{s}=\left(v_{j}, v_{k}\right) .
\end{aligned}
$$

Let $T=\left(e_{1}, \cdots, e_{s-2}\right)$. Then the term corresponding to $P, Q$ and $R$ in the sum $\sum_{j}\left(\mathbf{A}_{s-1}\right)_{i, j} \mathbf{A}_{j, k}$ is $u^{b c(T)}, u^{b c(T)}$ and $u^{b c(T)+1}$, respectively. While, the term corresponding to $P, Q$ and $R$ in $\left(\mathbf{A}_{s}\right)_{i, k}$ is $u^{b c(T)}, u^{b c(T)+1}$ and $u^{b c(T)+2}$, respectively. Thus,

$$
\left(\mathbf{A}_{s}\right)_{i, k}=\sum_{j}\left(\mathbf{A}_{s-1}\right)_{i, j} \mathbf{A}_{j, k}+(u-1)\left(\mathbf{A}_{s-2}\right)_{i, k} q_{k}+\left(u^{2}-u\right)\left(\mathbf{A}_{s-2}\right)_{i, k},
$$

where $q_{k}=\operatorname{deg} v_{k}-1$. Therefore, the result follows. Q.E.D.

For $s \geq 1$, let $\mathcal{C}_{s}^{\text {tail }}$ be the set of all closed paths of length $s$ with tails in $G$, and

$$
a_{s}=\lim _{n \rightarrow \infty} \frac{1}{\left|V\left(K_{n}\right)\right|} \sum_{x \in V\left(K_{n}\right)}\left\{u^{b c(C)} \mid C \in \mathcal{C}_{s}^{\text {tail }} \text { and } o(C)=x\right\} .
$$

Then $a_{1}=0$.

Lemma 2 1. For $s \in \mathbf{N}, a_{s}$ exists and is finite.

2.

$$
a_{s}=\operatorname{Tr}_{\mathcal{I}}\left[(\mathbf{Q}-(1-2 u) \mathbf{I}) \mathbf{A}_{s-2}\right]+(1-u)^{2} a_{s-2} \text { for } s \geq 3 .
$$

Proof. 1: For $n \in \mathbf{N}$, let

$$
\Omega_{n}=V\left(K_{n}\right) \backslash B_{1}\left(\mathcal{F}_{\mathcal{I}}\left(K_{n}\right)\right), \Omega_{n}^{\prime}=V\left(K_{n}\right) \cap B_{1}\left(\mathcal{F}_{\mathcal{I}}\left(K_{n}\right)\right) .
$$

Then, for all $p \in \mathbf{N}$,

$$
V\left(K_{n+p}\right)=\cup_{\gamma \in \mathcal{I}(n, n+p)} \gamma \Omega_{n} \cup\left(\cup_{\gamma \in \mathcal{I}(n, n+p)} \gamma \Omega_{n}^{\prime}\right) .
$$

Let

$$
a_{s}(x)=\sum_{x \in V\left(K_{n}\right)}\left\{u^{b c(C)} \mid C \in \mathcal{C}_{s}^{\text {tail }} \text { and } o(C)=x\right\} .
$$


Then $a_{s}(x) \leq d^{s-1}$. Thus, by 1 and 3 of Proposition 1 , we have

$$
\begin{aligned}
& \left|\frac{1}{\left|V\left(K_{n+p}\right)\right|} \sum_{x \in V\left(K_{n+p}\right)} a_{s}(x)-\frac{1}{\left|V\left(K_{n}\right)\right|} \sum_{x \in V\left(K_{n}\right)} a_{s}(x)\right| \\
& \leq\left|\frac{|\mathcal{I}(n, n+p)|}{\left|V\left(K_{n+p}\right)\right|} \sum_{x \in \Omega_{n}} a_{s}(x)-\frac{1}{\left|V\left(K_{n}\right)\right|} \sum_{x \in V\left(K_{n}\right)} a_{s}(x)\right|+\frac{|\mathcal{I}(n, n+p)|}{\left|V\left(K_{n+p}\right)\right|} \sum_{x \in \Omega_{n}^{\prime}}\left|a_{s}(x)\right| \\
& \leq\left|\frac{|\mathcal{I}(n, n+p)|}{\left|V\left(K_{n+p}\right)\right|}-\frac{1}{\left|V\left(K_{n}\right)\right|}\right| \sum_{x \in V\left(K_{n}\right)}\left|a_{s}(x)\right|+2 \frac{|\mathcal{I}(n, n+p)|}{\left|V\left(K_{n+p}\right)\right|} \sum_{x \in B_{1}\left(\mathcal{F}_{\mathcal{I}}\left(K_{n}\right)\right)}\left|a_{s}(x)\right|
\end{aligned}
$$

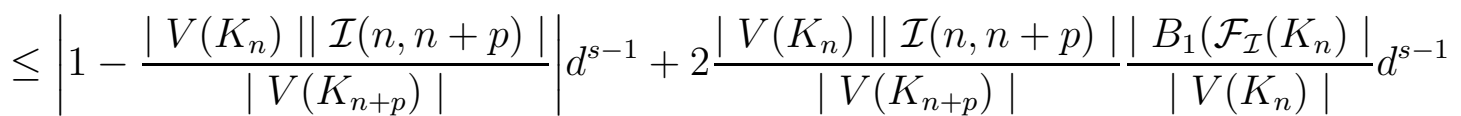

$$
\begin{aligned}
& \leq 2 \epsilon_{n}(d+1) d^{s-1}+2 \frac{\left|V\left(K_{n}\right)\right||\mathcal{I}(n, n+p)|\left|\mathcal{F}_{\mathcal{I}}\left(K_{n}\right)\right|(d+1)}{\left|V\left(K_{n+p}\right)\right|} d^{s-1} \\
& \leq 6 \epsilon_{n}(d+1) d^{s-1} \longrightarrow 0 \text { as } n \rightarrow \infty,
\end{aligned}
$$

where

$$
\epsilon_{n}=\frac{\left|\mathcal{F}_{\mathcal{I}}\left(K_{n}\right)\right|}{\left|V\left(K_{n}\right)\right|} \rightarrow 0 \text { as } n \rightarrow \infty
$$

2: At first, we have

$$
\begin{aligned}
a_{s} & =\lim _{n \rightarrow \infty} \frac{1}{\left|V\left(K_{n}\right)\right|} \sum_{v_{i} \in V\left(K_{n}\right)}\left\{u^{b c(C)} \mid C \in \mathcal{C}_{s}^{\text {tail }} \text { and } o(C)=v_{i}\right\} \\
& =\lim _{n \rightarrow \infty} \frac{1}{\left|V\left(K_{n}\right)\right|} \sum_{v_{i} \in V\left(K_{n}\right)} \sum_{\left(v_{i}, v_{j}\right) \in R(G)}\left\{u^{b c(C)} \mid C=\left(v_{i}, v_{j}, \ldots\right) \in \mathcal{C}_{s}^{\text {tail }}\right\} \\
& =\lim _{n \rightarrow \infty} \frac{1}{\left|V\left(K_{n}\right)\right|} \sum_{v_{j} \in V\left(K_{n}\right)} \sum_{\left(v_{i}, v_{j}\right) \in R(G)}\left\{u^{b c(C)} \mid C=\left(v_{i}, v_{j}, \ldots\right) \in \mathcal{C}_{s}^{\text {tail }}\right\}
\end{aligned}
$$

The third equality is proved as follows: Let

$$
\Omega=\left\{v \in V(G) \mid v \notin V\left(K_{n}\right), d\left(v, K_{n}\right)=1\right\} \subset B_{1}\left(\mathcal{F}_{\mathcal{I}}\left(K_{n}\right)\right) .
$$

Then we have

$$
\begin{aligned}
\frac{1}{\left|V\left(K_{n}\right)\right|} & \sum_{v_{i} \in V\left(K_{n}\right)} \sum_{\left(v_{i}, v_{j}\right) \in R(G)}\left\{u^{b c(C)} \mid C=\left(v_{i}, v_{j}, \ldots\right) \in \mathcal{C}_{s}^{\text {tail }}\right\} \\
= & \frac{1}{\left|V\left(K_{n}\right)\right|} \sum_{v_{j} \in V\left(K_{n}\right)} \sum_{\left(v_{i}, v_{j}\right) \in R(G)}\left\{u^{b c(C)} \mid C=\left(v_{i}, v_{j}, \ldots\right) \in \mathcal{C}_{s}^{\text {tail }}\right\} \\
& +\frac{1}{\left|V\left(K_{n}\right)\right|} \sum_{v_{j} \in \Omega} \sum_{\left(v_{i}, v_{j}\right) \in R(G), v_{i} \in V\left(K_{n}\right)}\left\{u^{b c(C)} \mid C=\left(v_{i}, v_{j}, \ldots\right) \in \mathcal{C}_{s}^{\text {tail }}\right\} \\
& -\frac{1}{\left|V\left(K_{n}\right)\right|} \sum_{v_{j} \in V\left(K_{n}\right)} \sum_{\left(v_{i}, v_{j}\right) \in R(G), v_{i} \in \Omega}\left\{u^{b c(C)} \mid C=\left(v_{i}, v_{j}, \ldots\right) \in \mathcal{C}_{s}^{\text {tail }}\right\} .
\end{aligned}
$$


But, we have

$$
\begin{gathered}
\left|\frac{1}{\left|V\left(K_{n}\right)\right|} \sum_{v_{j} \in \Omega} \sum_{\left(v_{i}, v_{j}\right) \in R(G), v_{i} \in V\left(K_{n}\right)}\left\{u^{b c(C)} \mid C=\left(v_{i}, v_{j}, \ldots\right) \in \mathcal{C}_{s}^{\text {tail }}\right\}\right| \\
\quad \leq \frac{1}{\left|V\left(K_{n}\right)\right|}\left|\mathcal{F}_{\mathcal{I}}\left(K_{n}\right)\right| d^{s-1} \longrightarrow 0
\end{gathered}
$$

and

$$
\begin{aligned}
\left|\frac{1}{\left|V\left(K_{n}\right)\right|} \sum_{v_{j} \in V\left(K_{n}\right)} \sum_{\left(v_{i}, v_{j}\right) \in R(G), v_{i} \in \Omega}\left\{u^{b c(C)} \mid C=\left(v_{i}, v_{j}, \ldots\right) \in \mathcal{C}_{s}^{\text {tail }}\right\}\right| \\
=\left|\frac{1}{\left|V\left(K_{n}\right)\right|} \sum_{v_{j} \in \mathcal{F}_{\mathcal{I}}\left(K_{n}\right)} \sum_{\left(v_{i}, v_{j}\right) \in R(G), v_{i} \in \Omega}\left\{u^{b c(C)} \mid C=\left(v_{i}, v_{j}, \ldots\right) \in \mathcal{C}_{s}^{\text {tail }}\right\}\right| \\
\leq \frac{1}{\left|V\left(K_{n}\right)\right|}\left|\mathcal{F}_{\mathcal{I}}\left(K_{n}\right)\right| d^{s-1} \longrightarrow 0 .
\end{aligned}
$$

Thus, the third equality holds.

We want to count closed paths of length $s$ with tails in $G$. The proof is an analogue of the proof of Lemma 2 in [21].

Let $s \geq 3$ and let $v_{j}$ be fixed. Furthermore, let $C=\left(v_{i}, v_{j}, v_{l}, \cdots, v_{r}, v_{j}, v_{i}\right)$ be any closed path of length $s$ with tails in $G$, and let $P=\left(v_{j}, v_{l}, \cdots, v_{r}, v_{j}\right)$.

Case 1. $P$ does not have a tail, i.e., $v_{l} \neq v_{r}$.

Then the closed path $C$ is divided into two types:

$$
\begin{aligned}
& C_{1}=\left(v_{i}, v_{j}, v_{l}, \cdots, v_{r}, v_{j}, v_{i}\right), v_{i} \neq v_{l} \text { and } v_{i} \neq v_{r}, \\
& C_{2}=\left(v_{i}, v_{j}, v_{i}, \cdots, v_{r}, v_{j}, v_{i}\right)\left(v_{l}=v_{i}\right) \\
& \quad \text { or }\left(v_{i}, v_{j}, v_{l}, \cdots, v_{i}, v_{j}, v_{i}\right)\left(v_{r}=v_{i}\right) .
\end{aligned}
$$

Case 2. $P$ has a tail, i.e., $v_{l}=v_{r}$.

Then the closed path $C$ is divided into two types:

$$
\begin{aligned}
& C_{3}=\left(v_{i}, v_{j}, v_{l}, \cdots, v_{l}, v_{j}, v_{i}\right), v_{i} \neq v_{l}, \\
& C_{4}=\left(v_{i}, v_{j}, v_{i}, \cdots, v_{i}, v_{j}, v_{i}\right), v_{i}=v_{l} .
\end{aligned}
$$

Now, we have

$$
u^{b c\left(C_{1}\right)}=u^{b c\left(C_{3}\right)}=u^{b c(P)}, u^{b c\left(C_{2}\right)}=u^{b c(P)+1}, u^{b c\left(C_{4}\right)}=u^{b c(P)+2} .
$$

Thus,

$$
\begin{aligned}
b_{j}= & \sum_{\left(v_{i}, v_{j}\right) \in R(G)}\left\{u^{b c(C)} \mid C \supset \text { tail },|C|=s, C=\left(v_{i}, v_{j}, \cdots\right)\right\} \\
= & \left(q_{j}-1\right) \sum\left\{u^{b c(P)} \mid P \not \supset \text { tail },|P|=s-2, P: v_{j}-\text { closed path }\right\} \\
& +2 u \sum\left\{u^{b c(P)} \mid P \not \supset \text { tail, }|P|=s-2, P: v_{j}-\text { closed path }\right\} \\
& +q_{j} \sum\left\{u^{b c(P)} \mid P \supset \text { tail, }|P|=s-2, P: v_{j}-\text { closed path }\right\} \\
& +u^{2} \sum\left\{u^{b c(P)} \mid P \supset \text { tail, }|P|=s-2, P: v_{j}-\text { closed path }\right\} .
\end{aligned}
$$


That is,

$$
\begin{aligned}
b_{j}= & \left(q_{j}-1\right) \sum\left\{u^{b c(P)}|| P \mid=s-2, P: v_{j} \text {-closed path }\right\} \\
& +2 u \sum\left\{u^{b c(P)} \mid P \not \supset \text { tail, }|P|=s-2, P: v_{j}-\text { closed path }\right\} \\
& +\left(1+u^{2}\right) \sum\left\{u^{b c(P)} \mid P \supset \text { tail, }|P|=s-2, P: v_{j} \text { - closed path }\right\} .
\end{aligned}
$$

Therefore, it follows that

$$
\begin{aligned}
a_{s}= & \lim _{n \rightarrow \infty} \frac{1}{\left|V\left(K_{n}\right)\right|} \sum_{v_{j} \in V\left(K_{n}\right)} b_{j} \\
= & \lim _{n \rightarrow \infty} \frac{1}{\left|V\left(K_{n}\right)\right|} \sum_{v_{j} \in V\left(K_{n}\right)}\left(\mathbf{Q}\left(v_{j}, v_{j}\right)-1\right) \sum\left\{u^{b c(P)}|| P \mid=s-2, P: v_{j}-\text { closed path }\right\} \\
& +2 u \lim _{n \rightarrow \infty} \frac{1}{\left|V\left(K_{n}\right)\right|} \sum_{v_{j}} \sum\left\{u^{b c(P)}|| P \mid=s-2, P: v_{j}-\text { closed path }\right\} \\
& +\left(1-2 u+u^{2}\right) \lim _{n \rightarrow \infty} \frac{1}{\left|V\left(K_{n}\right)\right|} \sum_{v_{j}} \sum\left\{u^{b c(P)} \mid P \supset\right. \text { tail, } \\
& \left.|P|=s-2, P: v_{j}-\text { closed path }\right\} .
\end{aligned}
$$

Hence,

$$
a_{s}=\operatorname{Tr}_{\mathcal{I}}\left[(\mathbf{Q}-\mathbf{I}) \mathbf{A}_{s-2}\right]+2 u \operatorname{Tr}_{\mathcal{I}}\left[\mathbf{A}_{s-2}\right]+(1-u)^{2} a_{s-2}
$$

Q.E.D.

For $m \geq 1$, let $\mathcal{C}_{m}$ be the set of all closed paths of length $s$ in $G$, and

$$
N_{m}=\lim _{n \rightarrow \infty} \frac{1}{\left|V\left(K_{n}\right)\right|} \sum\left\{u^{c b c(C)} \mid C \in \mathcal{C}_{m} \text { and } C \subset K_{n}\right\} .
$$

Lemma 3 1. For $m \in \mathbf{N}, N_{m}$ exists and is finite.

2. $N_{m}=\operatorname{Tr}_{\mathcal{I}}\left(\mathbf{A}_{m}\right)-(1-u) a_{m}$.

Proof. 1: At first, we have

$$
N_{m}=\lim _{n \rightarrow \infty} \frac{1}{\left|V\left(K_{n}\right)\right|} \sum\left\{u^{c b c(C)} \mid C \in \mathcal{C}_{m} \text { and } o(C)=v \in V\left(K_{n}\right)\right\} .
$$


For, by 2 of Proposition 1,

$$
\begin{aligned}
0 \leq & \mid \frac{1}{\left|V\left(K_{n}\right)\right|}\left(\sum\left\{u^{c b c(C)} \mid C \in \mathcal{C}_{m} \text { and } o(C)=v \in V\left(K_{n}\right)\right\}\right. \\
& \left.-\sum\left\{u^{c b c(C)} \mid C \in \mathcal{C}_{m} \text { and } C \subset K_{n}\right\}\right) \mid \\
= & \frac{1}{\left|V\left(K_{n}\right)\right|} \mid \sum\left\{u^{c b c(C)} \mid C \in \mathcal{C}_{m}, o(C)=v \in V\left(K_{n}\right) \text { and } C \not \subset K_{n}\right\} \mid \\
\leq & \frac{1}{\left|V\left(K_{n}\right)\right|}\left|\sum\left\{u^{b c(C)} \mid C \in \mathcal{C}_{m}, o(C)=v \in B_{m}\left(\mathcal{F}_{\mathcal{I}}\left(K_{n}\right)\right)\right\}\right| \\
= & \frac{1}{\left|V\left(K_{n}\right)\right|}\left|\sum_{v \in B_{m}\left(\mathcal{F}_{\mathcal{I}}\left(K_{n}\right)\right)} \mathbf{A}_{m}(v \cdot v)\right| \\
\leq & \frac{1}{\left|V\left(K_{n}\right)\right|}\left|\operatorname{Tr}\left(P\left(B_{m}\left(\mathcal{F}_{\mathcal{I}}\left(K_{n}\right)\right)\right) \mathbf{A}_{m}\right)\right| \\
\leq & \left\|\mathbf{A}_{m}\right\| \frac{\left|B_{m}\left(\mathcal{F}_{\mathcal{I}}\left(K_{n}\right)\right)\right|}{\left|V\left(K_{n}\right)\right|} \\
\leq & \left\|\mathbf{A}_{m}\right\|(d+1)^{m} \frac{\left|\mathcal{F}_{\mathcal{I}}\left(K_{n}\right)\right|}{\left|V\left(K_{n}\right)\right|} \longrightarrow 0
\end{aligned}
$$

if $n \rightarrow \infty$.

Furthermore, the existence of

$$
\lim _{n \rightarrow \infty} \frac{1}{\left|V\left(K_{n}\right)\right|} \sum\left\{u^{c b c(C)} \mid C \in \mathcal{C}_{m} \text { and } o(C)=v \in V\left(K_{n}\right)\right\}
$$

is proved as 1 of Lemma 2.

Therefore, it follows that

$$
\begin{aligned}
N_{m}= & \lim _{n \rightarrow \infty} \frac{1}{\left|V\left(K_{n}\right)\right|} \sum\left\{u^{c b c(C)} \mid C \in \mathcal{C}_{m} \text { and } o(C)=v \in V\left(K_{n}\right)\right\} \\
= & \lim _{n \rightarrow \infty} \frac{1}{\left|V\left(K_{n}\right)\right|} \sum\left\{u^{b c(C)} \mid C \in \mathcal{C}_{m}^{\text {nontail }} \text { and } o(C)=v \in V\left(K_{n}\right)\right\} \\
& +\lim _{n \rightarrow \infty} \frac{1}{\left|V\left(K_{n}\right)\right|} \sum\left\{u^{c b c(C)} \mid C \in \mathcal{C}_{m}^{\text {tail }} \text { and } o(C)=v \in V\left(K_{n}\right)\right\} \\
= & \lim _{n \rightarrow \infty} \frac{1}{\left|V\left(K_{n}\right)\right|} \sum_{v \in V\left(K_{n}\right)} \mathbf{A}_{m}(v \cdot v) \\
& -\lim _{n \rightarrow \infty} \frac{1}{\left|V\left(K_{n}\right)\right|} \sum_{v \in V\left(K_{n}\right)} \sum\left\{u^{b c(C)}-u^{c b c(C)} \mid C \in \mathcal{C}_{m}^{\text {tail }} \text { and } o(C)=v \in V\left(K_{n}\right)\right\} .
\end{aligned}
$$

Hence, since $c b c(C)=b c(C)+1$ for each closed path $C$ of length $s$ with tails, we have

$$
N_{m}=\operatorname{Tr}_{\mathcal{I}}\left(\mathbf{A}_{m}\right)-(1-u) a_{m}
$$

Q.E.D. 


\section{The Bartholdi zeta function of a fractal graph}

We define the notion of $\mathcal{I}$-equivalence between cycles. Let $G$ be a connected fractal graph. Furthermore, let $\left\{K_{n}\right\}$ be an amenable exhaustion of $G$ such that satisfies the conditions (i) and (ii) in the definition of a fractal graph. For $[C],[D] \in \mathcal{K},[C]$ and $[D]$ are called $\mathcal{I}$-equivalent, denoted $[C] \sim \sim_{\mathcal{I}}[D]$, if there exista a local isomorphism $\gamma \in \mathcal{I}$ such that $D=\gamma(C)$. We denote by $[C]_{\mathcal{I}}$ the set of $\mathcal{I}$-equivalent class containing $[C]$. Note that $[C] \in[C]_{\mathcal{I}}$. Let $[\mathcal{K}]_{\mathcal{I}}$ and $[\mathcal{P} \mathcal{K}]_{\mathcal{I}}$ be the set of $\mathcal{I}$-equivalence classes of $\mathcal{K}$ and $\mathcal{P} \mathcal{K}$, respectively.

For $[C] \in \mathcal{K}$, the size $s(C) \in \mathbf{N}$ of $[C]$ is the least $m \in \mathbf{N}$ such that $C \subset \gamma\left(K_{m}\right)$ for some local isomorphism $\gamma \in \mathcal{I}(m)$. Furthermore, the effective length $\ell(C) \in \mathbf{N}$ of $[C]$ is the length of the primitive closed path $D$ underlying $C$, i.e., such that $C=D^{p}$ for some $p \in \mathbf{N}$. The average multiplicity $\mu(C)$ of $[C]$ is the number in $[0, \infty)$ given by

$$
\lim _{n \rightarrow \infty} \frac{|\mathcal{I}(s(C), n)|}{\left|V\left(K_{n}\right)\right|}
$$

Lemma 4 1. Let $[C] \in \mathcal{K}$. Then the following limit exists and is finite:

$$
\lim _{n \rightarrow \infty} \frac{|\mathcal{I}(s(C), n)|}{\left|V\left(K_{n}\right)\right|}
$$

2. $s(C), \ell(C), \mu(C)$ only depend on $[C]_{\mathcal{I}} \in[\mathcal{K}]_{\mathcal{I}}$. Furthermore, if $C=D^{k}$ for some $[D] \in \mathcal{P} \mathcal{K}, k \in \mathbf{N}$, then $s(C)=s(D), \ell(C)=\ell(D), \mu(C)=\mu(D)$.

3. For $m \in \mathbf{N}$,

$$
N_{m}=\sum_{[C]_{\mathcal{I}} \in\left[\mathcal{K}_{m}\right]_{\mathcal{I}}} \mu(C) \ell(C) u^{c b c(C)}
$$

Proof. 1: At first, we have

$$
|\mathcal{I}(s(C), n+1)|=|\mathcal{I}(s(C), n)||\mathcal{I}(n, n+1)|
$$

for any $n \geq s(C)$. By 2 and 3 of Proposition 1, we obtain

$$
\begin{aligned}
\left|\frac{|\mathcal{I}(s(C), n)|}{\left|V\left(K_{n}\right)\right|}-\frac{|\mathcal{I}(s(C), n+p)|}{\left|V\left(K_{n+p}\right)\right|}\right| & =\frac{|\mathcal{I}(s(C), n)|}{\left|V\left(K_{n}\right)\right|}\left|1-\frac{\left|V\left(K_{n}\right)\right||\mathcal{I}(n, n+p)| \mid}{\left|V\left(K_{n+p}\right)\right|}\right| \\
& \leq \frac{1}{\left|\Omega_{n, 1}\right|} 2 \epsilon_{n}(d+1) .
\end{aligned}
$$

Furthermore,

$$
\frac{|\mathcal{I}(s(C), n+1)|}{\left|V\left(K_{n+1}\right)\right|}=\frac{|\mathcal{I}(s(C), n)|}{\left|V\left(K_{n}\right)\right|} \frac{\left|V\left(K_{n}\right)\right||\mathcal{I}(n, n+1)|}{\left|V\left(K_{n+1}\right)\right|} \geq \frac{|\mathcal{I}(s(C), n)|}{\left|V\left(K_{n}\right)\right|},
$$

and so the limit is monotone. 
2: Clear.

3: We have

$$
\begin{aligned}
N_{m} & =\lim _{n \rightarrow \infty} \frac{1}{\left|V\left(K_{n}\right)\right|} \sum\left\{u^{c b c(C)} \mid C \in \mathcal{C}_{m} \text { and } C \subset K_{n}\right\} \\
& =\lim _{n \rightarrow \infty} \sum_{[C]_{\mathcal{I}} \in\left[\mathcal{K}_{m}\right]_{\mathcal{I}}} \frac{1}{\left|V\left(K_{n}\right)\right|} \sum\left\{u^{c b c(D)} \mid D \in \mathcal{C}_{m},[D] \sim{ }_{\mathcal{I}}[C], D \subset K_{n}\right\} \\
& =\lim _{n \rightarrow \infty} \sum_{[C]_{\mathcal{I}} \in\left[\mathcal{K}_{m}\right]_{\mathcal{I}}} \frac{1}{\left|V\left(K_{n}\right)\right|} u^{c b c(C)} \ell(C)|\mathcal{I}(s(C), n)| \\
& =\sum_{[C]_{\mathcal{I}} \in\left[\mathcal{K}_{m}\right]_{\mathcal{I}}} u^{c b c(C)} \ell(C) \mu(C) .
\end{aligned}
$$

Q.E.D.

We define the Bartholdi zeta function of a fractal graph as follows:

$$
\zeta_{G, \mathcal{I}}(u, t)=\prod_{[C]_{\mathcal{I}} \in[\mathcal{P} \mathcal{K}]_{\mathcal{I}}}\left(1-u^{c b c(C)} t^{|C|}\right)^{-\mu(C)},
$$

where $u, t \in \mathbf{C}$ are sufficiently small such that the infinite product converges, and $u>0$.

\section{Lemma 5}

$$
\frac{\partial}{\partial t} \log \zeta_{G, \mathcal{I}}(u, t)=t^{-1} \sum_{s \geq 1} N_{s} t^{s}
$$

Proof. Since

$$
\begin{aligned}
\log \zeta_{G, \mathcal{I}}(u, t) & =-\mu(C) \sum_{[C]_{\mathcal{I}} \in[\mathcal{P} \mathcal{K}]_{\mathcal{I}}} \log \left(1-u^{c b c(C)} t^{|C|}\right) \\
& =\mu(C) \sum_{[C]_{\mathcal{I}} \in[\mathcal{P} \mathcal{K}]_{\mathcal{I}}} \sum_{s=1}^{\infty} \frac{1}{s} u^{c b c(C) s} t^{|C| s},
\end{aligned}
$$

we have

$$
\begin{aligned}
\frac{\partial}{\partial t} \log \zeta_{G, \mathcal{I}}(u, t) & =t^{-1} \sum_{[C]_{\mathcal{I}} \in[\mathcal{P} \mathcal{K}]_{\mathcal{I}}} \sum_{s=1}^{\infty} \mu(C)|C| u^{c b c(C) s} t^{|C| s} \\
& =t^{-1} \sum_{s=1}^{\infty} \sum_{[C]_{\mathcal{I}} \in[\mathcal{P} \mathcal{K}]_{\mathcal{I}}} \mu(C)|C| u^{c b c(C) s} t^{|C| s} \\
& =t^{-1} \sum_{\left[C_{1}\right]_{\mathcal{I}} \in[\mathcal{K}]_{\mathcal{I}}} \mu\left(C_{1}\right) \ell\left(C_{1}\right) u^{c b c\left(C_{1}\right)} t^{\left|C_{1}\right|}
\end{aligned}
$$

Note that $c b c\left(C^{s}\right)=c b c(C) s$. The third equality is obtained by the fact that each closed path of $G$ is a multiple of some primitive closed path of $G$. 
Therefore, by Lemma 4, it follows that

$$
\frac{\partial}{\partial t} \log \zeta_{G, \Gamma}(u, t)=t^{-1} \sum_{s \geq 1} N_{s} t^{s}
$$

Q.E.D.

\section{Analytic determinants for von Neumann algebras with a finite trace}

In an excellent paper [6], Fuglede and Kadison defined a positive-valued determinant for von Neumann algebras with trivial center and finite trace. For an invertible operator $A$ with polar decomposition $A=U H$, the Fuglede-Kadison determinant of $A$ is defined by

$$
\operatorname{Det}(A)=\exp \circ \tau \circ \log H,
$$

where $\log H$ may be defined via the functional calculus.

Guido, Isola and Lapidus [9] extended the Fuglede-Kadison determinant to a determinant which is an analytic function. Let $(\mathcal{A}, \tau)$ be a von Neumann algebra with a finite trace. Then, for $A \in \mathcal{A}$, let

$$
\operatorname{det}_{\tau}(A)=\exp \circ \tau \circ \log A
$$

where

$$
\log (A):=\frac{1}{2 \pi i} \int_{\Gamma} \log \lambda(\lambda-A)^{-1} d \lambda
$$

and $\Gamma$ is the boundary of a connected, simply connected region $\Omega$ containing the spectrum $\sigma(A)$ of $A$. Then the following lemma holds(see [9, Lemma 5]).

Lemma 6 (Guido, Isola and Lapidus) Let $\mathcal{A}, \Omega, \Gamma$ be as above, and $\phi, \psi$ two branches of the logarithm such that both domains contain $\Omega$. Then

$$
\exp \circ \tau \circ \phi(A)=\exp \circ \tau \circ \psi(A)
$$

Next, we consider a determinant on some subset of $\mathcal{A}$. Let $(\mathcal{A}, \tau)$ be a von Neumann algebra with a finite trace, and $\mathcal{A}_{0}=\{A \in \mathcal{A} \mid 0 \notin \operatorname{conv} \sigma(A)\}$. For any $A \in \mathcal{A}_{0}$, we set

$$
\operatorname{det}_{\tau}(A)=\exp \circ \tau \circ\left(\frac{1}{2 \pi i} \int_{\Gamma} \log \lambda(\lambda-A)^{-1} d \lambda\right),
$$

where $\Gamma$ is the boundary of a connected, simply connected region $\Omega$ containing the spectrum conv $\sigma(A)$, and $\log$ is a branch of the logarithm whose domain contains $\Omega$. Then the above determinant is well-defined and analytic on $\mathcal{A}_{0}$ (see [9, Corollary 5.3]). Furthermore, Guido, Isola and Lapidus [9] showed that $\operatorname{det}_{\tau}$ has the following properties. 
Proposition 2 (Guido, Isola and Lapidus) Let $(\mathcal{A}, \tau)$ be a von Neumann algebra with a finite trace, $A \in \mathcal{A}_{0}$. Then

1. $\operatorname{det}_{\tau}(z A)=z \operatorname{det}_{\tau}(A)$ for any $z \in \mathbf{C} \backslash\{0\}$.

2. If $A$ is normal, and $A=U H$ is its polar decomposition, then

$$
\operatorname{det}_{\tau}(A)=\operatorname{det}_{\tau}(U) \operatorname{det}_{\tau}(H) .
$$

3. If $A$ is positive, then $\operatorname{det}_{\tau}(A)=\operatorname{Det}(A)$, where $\operatorname{Det}(A)$ is the Fuglede-Kadison determinant of $A$.

\section{A determinant expression}

In this section, we consider the following determinant:

$$
\operatorname{det}_{\mathcal{I}}(A)=\exp \circ \operatorname{Tr}_{\mathcal{I}} \circ \log A
$$

for $A \in \mathcal{A}(G)$.

In $\left(\sum_{s>0} \mathbf{A}_{s} t^{s}\right)\left(\mathbf{I}-t \mathbf{A}_{1}+(1-u)(\mathbf{Q}+u \mathbf{I}) t^{2}\right)$, the coefficient of $t^{s}$ for any $s \geq 3$ is 0 by the second formula of Lemma 1. Furthermore, by the first formula of Lemma 1, we have

$$
\left(\sum_{s \geq 0} \mathbf{A}_{s} t^{s}\right)\left(\mathbf{I}-t \mathbf{A}_{1}+(1-u)(\mathbf{Q}+u \mathbf{I}) t^{2}\right)=\left(1-(1-u)^{2} t^{2}\right) \mathbf{I} .
$$

Since $\left(1-(1-u)^{2} t^{2}\right)^{-1}=\sum_{j \geq 0}(1-u)^{2 j} t^{2 j}$,

$$
\begin{aligned}
\mathbf{I} & =\left(\sum_{k \geq 0} \mathbf{A}_{k} t^{k}\right)\left(\sum_{j \geq 0}(1-u)^{2 j} t^{2 j}\right)\left(\mathbf{I}-t \mathbf{A}_{1}+(1-u)(\mathbf{Q}+u \mathbf{I}) t^{2}\right) \\
& =\left(\sum_{s \geq 0} \sum_{j=0}^{[s / 2]} \mathbf{A}_{s-2 j}(1-u)^{2 j} t^{s}\right)\left(\mathbf{I}-t \mathbf{A}_{1}+(1-u)(\mathbf{Q}+u \mathbf{I}) t^{2}\right) .
\end{aligned}
$$

By Lemmas 2 and 3, we have

$N_{s}=\operatorname{Tr}_{\mathcal{I}}\left[\mathbf{A}_{s}-(1-u)^{-1}(\mathbf{Q}-(1-2 u) \mathbf{I}) \sum_{j=1}^{[(s-1) / 2]}(1-u)^{2 j} \mathbf{A}_{s-2 j}\right]- \begin{cases}0 & \text { if } s \text { is odd } \\ (1-u)^{s-1} a_{2} & \text { if } s \text { is even. }\end{cases}$

for $s \geq 3$. Furthermore, $N_{1}=\operatorname{Tr}_{\mathcal{I}} \mathbf{A}_{1}=0$, and

$$
\begin{aligned}
N_{2} & =\operatorname{Tr}_{\mathcal{I}} \mathbf{A}_{2}-(1-u) a_{2}=\lim _{n \rightarrow \infty} \frac{2 u\left|E\left(K_{n}\right)\right|}{\left|V\left(K_{n}\right)\right|}-(1-u) \lim _{n \rightarrow \infty} \frac{2 u\left|E\left(K_{n}\right)\right|}{\left|V\left(K_{n}\right)\right|} \\
& =2 u^{2} \lim _{n \rightarrow \infty} \frac{\left|E\left(K_{n}\right)\right|}{\left|V\left(K_{n}\right)\right|} .
\end{aligned}
$$


Next, set

$$
\begin{aligned}
\mathbf{N}_{s}^{*} & =\mathbf{A}_{s}-(1-u)^{-1}(\mathbf{Q}-(1-2 u) \mathbf{I}) \sum_{j=1}^{[s / 2]}(1-u)^{2 j} \mathbf{A}_{s-2 j} \\
& =\mathbf{A}_{s}+(1-u)^{-1}(\mathbf{Q}-(1-2 u) \mathbf{I}) \mathbf{A}_{s}-(1-u)^{-1}(\mathbf{Q}-(1-2 u) \mathbf{I}) \sum_{j=0}^{[s / 2]}(1-u)^{2 j} \mathbf{A}_{s-2 j} .
\end{aligned}
$$

Then (2) and (3) imply that

$$
\begin{aligned}
& \left(\sum_{s \geq 0} \mathbf{N}_{s}^{*} t^{s}\right)\left(\mathbf{I}-t \mathbf{A}_{1}+(1-u)(\mathbf{Q}+u \mathbf{I}) t^{2}\right) \\
& \quad=\left(\mathbf{I}+(1-u)^{-1}(\mathbf{Q}-(1-2 u) \mathbf{I})\right)\left(1-(1-u)^{2} t^{2}\right) \mathbf{I}-(1-u)^{-1}(\mathbf{Q}-(1-2 u) \mathbf{I}) \\
& \quad=\left(1-(1-u)^{2} t^{2}\right) \mathbf{I}-(1-u) t^{2}(\mathbf{Q}-(1-2 u) \mathbf{I}) .
\end{aligned}
$$

Since $\mathbf{N}_{0}^{*}=\mathbf{A}_{0}=\mathbf{I}_{n}$

$$
\begin{aligned}
& \left(\sum_{s \geq 1} \mathbf{N}_{s}^{*} t^{s}\right)\left(\mathbf{I}-t \mathbf{A}_{1}+(1-u)(\mathbf{Q}+u \mathbf{I}) t^{2}\right) \\
& \quad=\left(1-(1-u)^{2} t^{2}\right) \mathbf{I}-(1-u) t^{2}(\mathbf{Q}-(1-2 u) \mathbf{I})-\left(\mathbf{I}-t \mathbf{A}_{1}+(1-u)(\mathbf{Q}+u \mathbf{I}) t^{2}\right) \\
& \quad=t \mathbf{A}_{1}-2(1-u)(\mathbf{Q}+u \mathbf{I}) t^{2}
\end{aligned}
$$

Therefore it follows that

$$
\sum_{s \geq 1} \mathbf{N}_{s}^{*} t^{s}=\left(t \mathbf{A}_{1}-2(1-u)(\mathbf{Q}+u \mathbf{I}) t^{2}\right)\left(\mathbf{I}-t \mathbf{A}_{1}+(1-u)(\mathbf{Q}+u \mathbf{I}) t^{2}\right)^{-1}
$$

Lemma 7 Let $f: t \in B_{\epsilon}=\{t \in \mathbf{C}|| t \mid<\epsilon\} \mapsto f(u, t) \in \mathcal{A}(G)$ be a $\mathbf{C}^{1}$-function, $f(0,0)=0$, and $\|f(u, t)\|<1$ for all $t \in B_{\epsilon}$, where the absolute value of $u \in \mathbf{C}$ is suffiently small. Then

$$
\operatorname{Tr}_{\mathcal{I}}\left(-\frac{\partial}{\partial t} \log (\mathbf{I}-f(u, t))\right)=\operatorname{Tr}_{\mathcal{I}}\left(\frac{\partial}{\partial t} f(u, t)(\mathbf{I}-f(u, t))^{-1}\right) .
$$

Proof. At first, we have

$$
-\log (\mathbf{I}-f(u . t))=\sum_{n \geq 1} \frac{1}{n} f(u, t)^{n} .
$$

Then, the above converges in operator norm, uniformly on compact subsets of $B_{\epsilon}$, and \| $f(u, t) \|<1$ for all $t \in B_{\epsilon}$. Furthermore,

$$
\frac{\partial}{\partial t} f(u, t)^{n}=\sum_{j=0}^{n-1} f(u, t)^{j} \frac{\partial}{\partial t} f(u, t) f(u, t)^{n-j-1} .
$$


Therefore, we have

$$
\left.-\frac{\partial}{\partial t} \log (\mathbf{I}-f(u, t))\right)=\sum_{n \geq 1} \sum_{j=0}^{n-1} \frac{1}{n} f(u, t)^{j} \frac{\partial}{\partial t} f(u, t) f(u, t)^{n-j-1},
$$

and so

$$
\begin{aligned}
\operatorname{Tr}_{\mathcal{I}}\left(-\frac{\partial}{\partial t} \log (\mathbf{I}-f(u, t))\right) & =\sum_{n \geq 1} \frac{1}{n} \sum_{j=0}^{n-1} \operatorname{Tr}_{\mathcal{I}}\left(f(u, t)^{j} \frac{\partial}{\partial t} f(u, t) f(u, t)^{n-j-1}\right) \\
& =\sum_{n \geq 1} \operatorname{Tr}_{\mathcal{I}}\left(f(u, t)^{n-1} j \frac{\partial}{\partial t} f(u, t)\right) \\
& =\operatorname{Tr}_{\mathcal{I}}\left(\frac{\partial}{\partial t} f(u, t)(\mathbf{I}-f(u, t))^{-1}\right) .
\end{aligned}
$$

Q.E.D.

We state the average Euler-Poincaré characteristic of a fractal graph(see [11]).

Lemma 8 (Guido, Isola and Lapidus) The following limit exists and is finite:

$$
\chi_{a v}(G):=\lim _{n \rightarrow \infty} \frac{\chi\left(K_{n}\right)}{\left|V\left(K_{n}\right)\right|}=-\frac{1}{2} \operatorname{Tr}_{\mathcal{I}}(\mathbf{Q}-\mathbf{I})
$$

where $\chi\left(K_{n}\right)=\left|V\left(K_{n}\right)\right|-\left|E\left(K_{n}\right)\right|$.

\section{Theorem 5}

$$
\zeta_{G, \mathcal{I}}(u, t)^{-1}=\left(1-(1-u)^{2} t^{2}\right)^{-\chi_{a v}(G)} \operatorname{det}_{\mathcal{I}}\left(\mathbf{I}-t \mathbf{A}(G)+(1-u)(\mathbf{D}-(1-u) \mathbf{I}) t^{2}\right) .
$$

Proof. By Lemma 7, we have

$$
\operatorname{Tr}_{\mathcal{I}}\left(\sum_{s \geq 1} \mathbf{N}_{s}^{*} t^{s}\right)=\operatorname{Tr}_{\mathcal{I}}\left(-t \frac{\partial}{\partial t} \log \left(\mathbf{I}-t \mathbf{A}_{1}+(1-u)(\mathbf{Q}+u \mathbf{I}) t^{2}\right)\right) .
$$

By Lemma 8, we have

$$
a_{2}=u \lim _{n \rightarrow \infty} \frac{2\left|E\left(K_{n}\right)\right|}{\left|V\left(K_{n}\right)\right|}=u \operatorname{Tr}_{\mathcal{I}}(\mathbf{Q}+\mathbf{I})
$$

If $s$ is odd, then $\operatorname{Tr}_{\mathcal{I}}\left(N_{s}^{*}\right)=N_{s}$. Otherwise, we have

$$
\begin{aligned}
\operatorname{Tr}_{\mathcal{I}}\left(\mathbf{N}_{s}^{*}\right) & =N_{s}-(1-u)^{s-1} \operatorname{Tr}_{\mathcal{I}}(\mathbf{Q}-(1-2 u) \mathbf{I})+(1-u)^{s-1} a_{2} \\
& =N_{s}-(1-u)^{s-1} \operatorname{Tr}_{\mathcal{I}}(\mathbf{Q}-(1-2 u) \mathbf{I}-u(\mathbf{Q}+\mathbf{I})) \\
& =N_{s}-(1-u)^{s} \operatorname{Tr}_{\mathcal{I}}(\mathbf{Q}-\mathbf{I}) .
\end{aligned}
$$


Thus, for $s \geq 1$, we have

$$
\operatorname{Tr}_{\mathcal{I}}\left(\mathbf{N}_{s}^{*}\right)=N_{s}- \begin{cases}0 & \text { if } s \text { is odd } \\ (1-u)^{s} \operatorname{Tr}_{\mathcal{I}}(\mathbf{Q}-\mathbf{I}) & \text { if } s \text { is even }\end{cases}
$$

Thus,

$$
\begin{aligned}
\operatorname{Tr}_{\mathcal{I}}\left(\sum_{s \geq 1} \mathbf{N}_{s}^{*} t^{s}\right) & =\sum_{s \geq 1} N_{s} t^{s}-\operatorname{Tr}_{\mathcal{I}}(\mathbf{Q}-\mathbf{I})\left(\sum_{j \geq 1}(1-u)^{2 j} t^{2 j}\right) \\
& =\sum_{s \geq 1} N_{s} t^{s}-\operatorname{Tr}_{\mathcal{I}}(\mathbf{Q}-\mathbf{I}) \frac{(1-u)^{2} t^{2}}{1-(1-u)^{2} t^{2}}
\end{aligned}
$$

i.e.,

$$
\sum_{s \geq 1} N_{s} t^{s}=\operatorname{Tr}_{\mathcal{I}}\left(\sum_{s \geq 1} \mathbf{N}_{s}^{*} t^{s}\right)+\operatorname{Tr}_{\mathcal{I}}(\mathbf{Q}-\mathbf{I}) \frac{(1-u)^{2} t^{2}}{1-(1-u)^{2} t^{2}}
$$

(1) implies that

$$
\begin{aligned}
& t \frac{\partial}{\partial t} \log \zeta_{G, \mathcal{I}}(u, t) \\
& \quad=\operatorname{Tr}_{\mathcal{I}}\left(-t \frac{\partial}{\partial t} \log \left(\mathbf{I}-t \mathbf{A}_{1}+(1-u)(\mathbf{Q}+u \mathbf{I}) t^{2}\right)\right)+\operatorname{Tr}_{\mathcal{I}}(\mathbf{Q}-\mathbf{I}) \frac{(1-u)^{2} t^{2}}{1-(1-u)^{2} t^{2}} \\
& \quad=\operatorname{Tr}_{\mathcal{I}}\left(-t \frac{\partial}{\partial t} \log \left(\mathbf{I}-t \mathbf{A}_{1}+(1-u)(\mathbf{Q}+u \mathbf{I}) t^{2}\right)\right)-t \frac{\partial}{\partial t} \log \left(1-(1-u)^{2} t^{2}\right)^{\operatorname{Tr}_{\mathcal{I}}(\mathbf{Q}-\mathbf{I}) / 2}
\end{aligned}
$$

Both functions are 0 at $t=0$, and so

$$
\log \zeta_{G, \mathcal{I}}(u, t)=-\operatorname{Tr}_{\mathcal{I}}\left(\log \left(\mathbf{I}-t \mathbf{A}_{1}+(1-u)(\mathbf{Q}+u \mathbf{I}) t^{2}\right)\right)-\log \left(1-(1-u)^{2} t^{2}\right)^{\operatorname{Tr}_{\mathcal{I}}(\mathbf{Q}-\mathbf{I}) / 2}
$$

Hence the equality $\operatorname{Tr}_{\mathcal{I}}(\log (\mathbf{I}-\mathbf{B}))=\log \operatorname{det}_{\mathcal{I}}(\mathbf{I}-\mathbf{B})$ and Lemma 8 implies that

$$
\zeta_{G, \mathcal{I}}(u, t)=\left(1-(1-u)^{2} t^{2}\right)^{\chi_{a v}(G)} \operatorname{det}_{\mathcal{I}}\left(\mathbf{I}-t \mathbf{A}(G)+(1-u)(\mathbf{Q}+u \mathbf{I}) t^{2}\right)^{-1} .
$$

Q.E.D.

\section{Acknowledgment}

This work is supported by Grant-in-Aid for Science Research (C) in Japan. We would like to thank the referee for valuable comments and helpful suggestions. 


\section{References}

[1] M. T. Barlow, Heat kernels and sets with fractal structures, in "Heat kernels and Analysis on manifolds, Graphs, and Metric Spaces" (Paris, 2002), Contemp. Math. 338, Amer. Math. Soc., Providence, RI, 2003, pp. 11-40.

[2] L. Bartholdi, Counting paths in graphs, Enseign. Math. 45 (1999), 83-131.

[3] H. Bass, The Ihara-Selberg zeta function of a tree lattice, Internat. J. Math. 3 (1992), 717-797.

[4] B. Clair and S. Mokhtari-Sharghi, Zeta functions of discrete groups acting on trees, J. Algebra 237 (2001), 591-620.

[5] D. Foata and D. Zeilberger, A combinatorial proof of Bass's evaluations of the IharaSelberg zeta function for graphs, Trans. Amer. Math. Soc. 351 (1999), 2257-2274.

[6] B. Fuglede and R. Kadison, Determinant theory in infinite factors, Ann. Math. 55 (1952), 520-530.

[7] R. I. Grigorchuk, Symmetrical random walks on discrete groups, Adv. Probab. Rel. Top. (D. Griffeath ed.) vol. 6, M. Dekker 1980, 285-325, pp. 132-152.

[8] R. I. Grigorchuk and A. Żuk, The Ihara zeta function of infinite graphs, the KNS spectral measure and integrable maps, in : "Random Walk and Geometry", Proc. Workshop (Vienna, 2001), V. A. Kaimanovich et at., eds., de Gruyter, Berkin, 2004, pp. 141-180.

[9] D. Guido, T. Isola and M. L. Lapidus, Ihara zeta functions for periodic simple graphs, in $C^{*}$-algebras and elliptic theory II, p. 103-121. Editedby D. Burghelea, R. Melrose, A. Mishchenko, E. Troitsky, Trends in Mathematics, Birkhause Verlag, Basel, 2008.

[10] D. Guido, T. Isola and M. L. Lapidus, Ihara's zeta functions for periodic graphs and its approximation in the amenable case, J. Funct. Anal. 225 (2008), 1339-1361.

[11] D. Guido, T. Isola and M. L. Lapidus, A trace on fractal graphs and the Ihara zeta function, to appear in Trans. Amer. Math. Soc.

[12] B. M. Hambly and T. Kumagai, Heat kernel estimates for symmetric random walks on a class of fractal graphs and stability under rough isometries, in "Fractal Geometry and Applications: A jubilee of Benoit Mandelbrot", Proc. Sympos. Pure Math., 72, Part 2, Amer. Math. Soc., Providence, RI, 2004, pp. 233-259.

[13] K. Hashimoto, Zeta Functions of Finite Graphs and Representations of $p$-Adic Groups, Adv. Stud. Pure Math. Vol. 15, pp. 211-280, Academic Press, New York, 1989.

[14] Y. Ihara, On discrete subgroups of the two by two projective linear group over $p$-adic fields, J. Math. Soc. Japan 18 (1966), 219-235.

[15] M. Kotani and T. Sunada, Zeta functions of finite graphs, J. Math. Sci. U. Tokyo 7 (2000), 7-25.

[16] A. Lubotzky, R. Phillips and P. Sarnak, Ramanujan graphs, Combinatorica 8 (3) (1988), 261-277. 
[17] H. Mizuno and I. Sato, A new proof of Bartholdi's theorem, J. Algebraic Combin. 22 (2005), 259-271.

[18] B. Mohar, The spectrum of an infinite graph, Linear Algebra Appl. 48 (1982), 245256.

[19] B. Mohar and W. Woess, A survey on spectra of infinite graphs, Bull. London Math. Soc. 21 (1989), 209-234.

[20] J. -P. Serre, Trees, Springer-Verlag, New York, 1980.

[21] H. M. Stark and A. A. Terras, Zeta functions of finite graphs and coverings, Adv. Math. 121 (1996), 124-165.

[22] T. Sunada, L-Functions in Geometry and Some Applications, in Lecture Notes in Math., Vol. 1201, pp. 266-284, Springer-Verlag, New York, 1986.

[23] T. Sunada, Fundamental Groups and Laplacians(in Japanese), Kinokuniya, Tokyo, 1988.

[24] A. Terras, Fourier Analysis on Finite Groups and Applications, Cambridge Univ. Press, Cambridge (1999). 